
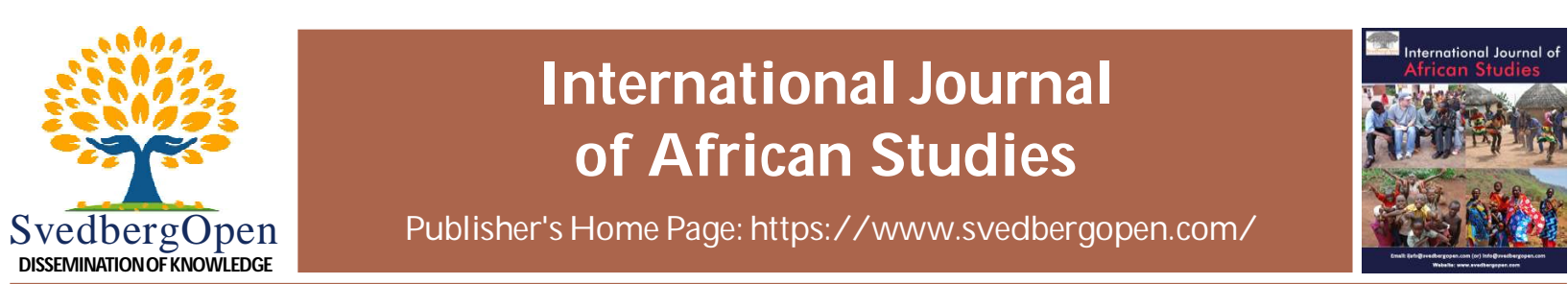

Research Paper

O pen A ccess

\title{
An investigation into Covid-19 related suicide risk factors in Malawi
}

\author{
Lloyd George Banda ${ }^{1 *}$ \\ ${ }^{1}$ Department of Political Science and Administrative Studies, Chancellor College, University of Malawi, Zomba, Malawi. \\ E-mail: ma-dev-12-20@cc.ac.mw
}

\section{Article Info}

Volume 1, Issue 2, June 2021

Received : 22 February 2021

Accepted : 15 May 2021

Published : 05 June 2021

doi: $10.51483 / I J A F R S .1 .2 .2021 .43-48$

\begin{abstract}
Malawi has witnessed a 57\% drastic upsurge in suicide cases since the outbreak of coronavirus pandemic in 2019. Of all these suicide cases, only one involved an outdoor Covid-19 patient. This research therefore sought to investigate the impact of Covid-19 among other risk factors on the suicide of the Covid-19 patient. The research incorporated both desk and field data collection and employed biological (nature), sociological (nurture) psychological (Thomas Joiner) theories in data analysis. There is no tangible evidence of the influence of Covid-19 critical adversity to the suicide incidence. The research paper concludes that committing suicide is about the things that people love so dearly without which they perceive an abnormal life. Finally, the paper recommends that labor laws should protect workers who are temporarily unavailable for an unknown period of time due to valid health reasons. It also advises employees to be conversant with the country's labor laws.
\end{abstract}

Keywords: Suicide, Risk factors, Covid-19, Labor laws, Love

(C) 2021 Lloyd George Banda. This is an open access article under the CC BY license (https://creativecommons.org/licenses/by/4.0/), which permits unrestricted use, distribution, and reproduction in any medium, provided you give appropriate credit to the original author(s) and the source, provide a link to the Creative Commons license, and indicate if changes were made.

\section{Introduction}

Malawi confirmed its first three cases of Covid-19 patients on April 2, 2020. This was followed with government attempt to impose a lockdown that was refuted by the courts considering the economic situation of the majority. Consequently, restrictions were placed on gathering and movements which negatively affected production activities and cut off supply chains. Suggestion is made therefore that a 57\% increase in suicide cases from 2019 to 2020 was as a result of the economic impacts of Covid-19 in the country. According to the Malawi police service report 116 people committed suicide in 2019. In 2020, a total of 182 people killed themselves, meaning that there has been a $57 \%$ increase. Reports also show that $92 \%$ of people who are committing suicide are men while the remaining $8 \%$ are women. On January 19, 2021, Malawi recorded the first Covid-19 patient suicide case.

Among the various mental disorders which tend to be closely related to suicide, depression is a particularly important risk factor. Many patients suffering from depression commit suicide never having had access to psychiatric care or proper treatment (Takahashi, 2001). Malawi - one of the poorest countries in Africa has a challenge stemming from the number of mental health experts and institutions. For example, Malawi has only one public mental hospital, and the few mental health experts in other hospitals are overburdened. This explains why many developing countries including Malawi faces an upsurge in suicide cases.

\footnotetext{
* Corresponding author:Lloyd George Banda, University of Malawi, Chancellor College, Department of Political and Administrative Studies. E-mail: ma-dev-12-20@cc.ac.mw
}

2710-4680/@ 2021. Lloyd George Banda. This is an open access article distributed under the Creative Commons Attribution License, which permits unrestricted use, distribution, and reproduction in any medium, provided the original work is properly cited. 


\subsection{Problem statement}

Studying causes of suicide has been one of the most intriguing area of research due complexities and sensitivity of the matter in data collection. Research findings rely on responses from interviewees because the dead persons cannot provide reasons behind taking their own lives. There are very few people who leave notes behind them explaining the reasons for taking their own lives. Technological advancement through checking of phones to find out the last persons the deceased talked to, has also proved to provide insufficient information on the real causes of suicide.

Suicide is the third leading cause of death among teenagers and young adults (Centers for Disease Control and Prevention, 2004). Depression as opposed to aggression has consistently been considered a risk factor in suicide, along with substance abuse, adverse life events, family history, a history of sexual abuse, troubled relationships, and difficulties with sexual identity (Compton and Berger 2001). However, most of these risk factors are aggregates studied from distance that ignores individual issues and personal characteristics. It is against this background that this research seeks to dwell on the first Covid-19 patient suicide case in Malawi in order to establish evidence-based conclusion on individual suicide risk factors.

\subsection{Background of the study}

The World Bank affirmed that for the first time in a generation, the quest to end poverty has suffered its worst setback. Many people who had barely escaped extreme poverty could be forced back into it by the convergence of Covid-19. A "nowcast" (preliminary estimate) for 2020, incorporating the effects of the Covid-19 pandemic, projects that an additional 88 million to 115 million people will be pushed into extreme poverty, bringing the total to between 703 and 729 million. This brings a large number of people into a situation they are not used to thereby creating depression, stress and anxiety. This is mainly because coronavirus is coming wave after wave leaving people hopeless and not knowing when the situation will normalize.

The outbreak of the novel coronavirus has led to a worst depression which vary from country to country. The supply chain has been cut in those countries' wort hit such as Western Europe, America and South Africa. The restriction of movements and various levels of lockdowns have led to shutting down and downsizing of many companies. This has led to various forms of financial challenges and has created a situation of anomie for people who lose their jobs, businesses and relationships. Since Durkheim's study on suicide in the $19^{\text {th }}$ century, increases in suicides during economic downturns are repeatedly observed. With the longevity of the existence of Covid-19 without remedy still obscure, the suicide-economic downturn nexus needs to be shrewdly investigated.

\subsection{Aims and objectives of the study}

This study seeks to investigate suicide risk factors at an individual level using the first Covid-19 patient suicide case in Malawi. The study will also examine whether depression is more fundamental than aggression in influencing decisions to commit suicide.

\section{Literature Review}

As many countries continually face new stay-at-home restrictions to curb the spread of Covid-19, there are concerns that rates of suicide may increase - or have already increased. Several factors underpin these concerns, including a deterioration in population mental capacity (depression, anxiety and/or stress) as a consequence of quarantine, isolation, economic hardship, and the unavailability of food and alcohol, the fear of getting the disease, a higher prevalence of reported thoughts and behaviors of self-harm among people with Covid-19, problems with accessing mental health services, and evidence suggesting that previous epidemics such as the 2003 SARS were also associated were associated with a rise in deaths by suicide (Zortea et al., 2020; Griffiths and Mamun, 2020). Supposition, however, is no replacement for evidence. Timely data on rates of suicide are vital and several studies reporting suicide trends have emerged more recently but the effect of Covid-19 on suicide should be interpreted with caution.

As of February 10, 2021, Malawi has experienced a single Covid-19 patient suicide case only in Blantyre. This is where this study dwells its attention to find out risk factors related to either depression or aggression. Malawi has recorded a $57 \%$ increase in suicides since the start of the coronavirus pandemic. The rest of suicide cases in Malawi during the Covid-19 era are attributed to financial challenges due to collapse of viability of small and medium enterprises. Most people in Malawi rely on day-to-day street trade and odd jobs stifled by movement restrictions (Benfica et al., 2018). This means the outbreak of coronavirus disrupted the normal ways of businesses and earning capacity of the majority of the people in the country. Yet around half of Malawi's 18.6 million population already live below the poverty 
line. According to the International Food Policy Research Institute, 1.6 million people have fallen below the poverty line category since the outbreak of the pandemic (Baulch et al., 2020).

US has been the country worst affected by Covid-19 with highest cases above 25 million and deaths almost half a million. Research shows that US has had increasing cases of suicide prior to the outbreak of coronavirus. For example, in 2017, suicide were the second causes of death for persons aged 10-24 (Curtin and Heron, 2019). Much as Covid-19 related suicide statistics are not readily available in the US, various reports indicated that rates of suicide ideation and attempts were higher during some months of 2020, as compared with 2019, but were not universally higher across this period. Months with significantly higher rates of suicide-related behaviors appear to correspond to times when Covidrelated stressors and community responses were heightened, indicating that youth experienced elevated distress during these periods (Hill et al., 2020).

Laanan (2015) conducted a related study on the impact of unemployment rate and the economic crisis on suicide mortality in Western European countries (2000-2010). This study used secondary data especially mortality statistics to model the yearly suicide rates using a quasi-pisson model, controlling for sex, age, country and a linear time trend to assess the unemployment-suicide association for each country. Statistical results showed that a significant $0.3 \%$ overall increase in suicide rate for a $10 \%$ increase in unemployment. The study concluded that unemployment and suicide rates are globally statistically associated in the investigated countries but its amplitude and sensitivity to the 'crisis-effect' vary across countries. However, this study did neither used primary data nor interviewed relations of those committing suicide to identify actual origins of suicide during financial crisis which is crucial in implementing efficient public health policies.

Another suicide study was conducted in Greece by Rachiotis et al. (2015). The objective of the study was to find out the effects of the Greek economic crisis especially fiscal austerity on suicide between 2003 to 2012. The study used age and sex specific suicide data provided by the Hellenic Statistical Authority. The analysis was divided into two; between 2003-2010 which was prior to the fiscal austerity measures and between 2011-2012 during the period of austerity. The results from regression models showed that the overall mean suicide rate rose by $35 \%$ between 2010 and 2012 , from 3.37 to 4.56 per 100,000 population. The study also found that each additional percentage point of unemployment was associated with a 0.19 per 100,000 population rise in suicides among men of working age. The study found a clear increase in suicides among persons of working age coinciding with fiscal austerity measures such that these findings corroborate concerns that increased suicide risk in Greece is a health hazard associated with fiscal austerity measures.

It is important to note that fiscal austerity means a cut in government expenditure in sectors like agriculture, health, construction or any other. This has a great potential of reducing incomes, creating unemployment and affecting accessibility and affordability of health and education. These may create depression or aggression which may lead to increase in suicide cases.

To the contrary, Islamic states have high rates of suicides but totally different in form from common trends. Asian countries lead by the Middle East are leading perpetrators of suicide bombings in the world. According to the Terrorism and Low Intensity Conflict Research Program at the Institute for National Security Studies (INSS), in 2016, 469 suicide bombings were carried out by 800 perpetrators in 28 countries (Kricheli et al., 2017). More specifically, the number of suicide bombings rose by $45 \%$ in the Middle East in 2016. However, suicide terrorists do not exhibit common characteristics of individuals bent on suicide. The desire to understand why and how individuals can decide to sacrifice themselves to kill others remains obscure. For many, this mentality is unfathomable. According to Harmon et al. (2018), suicide terrorist fall into a new category of suicide typology, that of fatalistic-altruistic suicides. This typology is defined as individuals who fit into Durkheim's (altruistic) and has suffered from long-term political and economic oppression and has no hope for their future (fatalistic).

According to WHO, close to 800,000 people die by suicide every year. Suicide is a serious global public health issue. It is among the top 20 leading causes of death worldwide, with more deaths due to suicide than to malaria, breast cancer, or war and homicide. The global age-standardized suicide rate was 10.5 per 100000 population for 2016. Rates varied between countries from less than five deaths by suicide per 100,000 to over 30 per 100,000. Globally, the majority of deaths by suicide occurred in low-and-middle-income countries (79\%), where most of the world's population lives $(84 \%)$. Regarding age, more than half $(52.1 \%)$ of global suicides occurred before the age of 45 years. Most adolescents who died by suicide (90\%) were from low- and middle-income countries where nearly $90 \%$ of the world's adolescents live. Much us there is no recent data on suicide related to coronavirus, there is a reverse in the trends with more suicide cases being reported in the western and eastern countries than in low- and middle-income countries. 


\section{Methodology}

\subsection{Sampling and data collection}

The research used both primary and secondary sources. The primary sources constituted both oral interviews and written information from documents. The paper also used important information from secondary written sources including journal articles, online books and articles. Oral interviews were conducted in Blantyre among the coworkers and neighbors of the diseased and in Mwanza among his childhood friends and relatives where he grew up. Mainly, the research targeted people who have had a direct relationship with the deceased for two or more years. Furthermore, the research uses nature and nurture and the interpersonal-psychological theory of suicidal behavior (Joiner, 2005) as the theoretical framework in the analysis of the suicide incident risk factors.

The research used structured interviews and life histories as research instruments. It should be noted that the procedure used to collect data was influenced by the research instruments used. Since a qualitative study is not always computable by arithmetic relations, the responses were categorized into various classes and I employed content analysis.

\subsection{Theoretical framework}

This section seeks to highlight and justify the theoretical approach used in this research and account for limitation and its remedies.

\subsubsection{Biological, sociological and psychological theories}

This research used both biological, sociological and psychological theories to analyze data. These theories are selected on the basis of their effect on altruism and self-interest decision making by people. Much or less as literature shows a great correlation between suicide and financial difficulties, the research does not employ any specific economic theory. This is because the economic well-being of people is taken into account in sociological theories as external (socialization) factors.

Nature and nurture: Nature is about what we think of as pre-wiring and is influenced by genetic inheritance and other biological factors. Nature is a biological concept borrowed in sociology in subject's quest to understand how people behave in societies. Edward O Wilson later dubbed this combination 'sociobiology'. According to Wilson, all human beings have certain genetically based instincts that influence behaviors and can be observed across cultures (Wilson, 1975).

On the other hand, nurture is a socialization concepts which takes into account the influence of external factors after conception, e.g., the product of exposure, life experiences and learning on an individual. This research will focus on family and peer groups as agents of socialization. As Kathy (2005) puts it, across all societies, the family is the first and the most important location of socialization.

Thomas Joiner suicide theories: The interpersonal-psychological theory of suicidal behavior (Joiner, 2005) proposes that an individual will not die by suicide unless s/he has both the desire to die by suicide and the ability to do so. The theory asserts that when people hold two specific psychological states in their minds simultaneously, and when they do so for long enough, they develop the desire for death. The two psychological states are perceived burdensomeness and $a$ sense of low belongingness or social alienation. In addition, the theory suggests that people are likely to surrender their lives if their adverse life experience is a burden to others. This research therefore, seeks to find out if testing positive of Covid-19 made the suicide culprit lose a sense of belonging due to alienation as argued by Kathy (2005) that isolation from human interaction can be quite damaging.

\section{Results and discussion}

The deceased committed suicide on the night of January 18, 2021, just a week after being diagnosed positive of Covid19. The research has found out that the deceased was a very reserved young man such that he rarely interacted with his coworkers beyond work related issues. One respondent who happened to be a close friend revealed that he needed to arrange a road trip if he wanted to syphon information from the deceased. As per psychologists, the suicide culprit will be referred as an introvert which is a genetically influenced instinct.

It is of little wonder that this research paper found more information from his last road trip companion in December 2020. During the 2020 Christmas holiday. The deceased accompanied a Zambian young lady, a student at the University of Malawi, to Zambia to see his girlfriend in Zambia. Two major findings are revealed from the conversation the two had on their way to Zambia; (1) the deceased loved the girlfriend dearly and the aim of the trip was to unveil himself to the girlfriend's parents; and (2) he loved his high school teaching job so much that he did not want to hunt another job. This 
reveals psychological excitement of the deceased about the things he loved. This is when he revealed that will never dare working with international organizations again due to short work contracts despite handsome pay. The trip revealed his strong desire to settle down at the high school he was working due to the indefinite work contract that he received.

Upon the return from Zambia his coworkers confessed that he became quieter, and isolated. It was later discovered that his girlfriend refused him to meet her parents in Zambia. However, his closest friend completely excludes this as a factor for his suicide because he was the one who terminated the relationship few days after returning to Malawi. Then later he was tested positive of coronavirus which sidelined him from work. This suggests an element of accumulating pain as argued by Thomas Joiner (2005) considering the resources the deceased worsted on his trip from Malawi to Zambia and the potentiality to lose his job.

Suggestion is made related to the leviathan work ethic at the high school in Blantyre where he was working. Interviewees revealed that three teachers who were diagnosed positive of coronavirus were still required to be conducting online lessons which might have been piling stress on these Covid-19 infected patients. When the first patient's sickness became critical and unable to conduct online lessons, the school replaced her with another teacher though there is no evidence whether the former was fired due to sickness but the new teacher responded that he was employed on a permanent basis with an indefinite work contract. Nevertheless, forcing workers to execute their duties when they are sick cannot be downplayed as a factor leading to depression and stress of losing their job.

The research also found out that the deceased was financially stable such that all respondents did not include financial difficulties as a risk factor to his death. For example, the deceased had a 3-toner lorry which he was using for hiring business and bought a saloon wagon just three weeks before surrendering his life. As per society standards, he was a rich person because his asset accumulation is rare among persons of his age groups.

The day before the night of the incident, the deceased received his young brother who had come to accompany the sister in sheering him up during his period of isolation. He was stronger enough to drive all the way to town to pick his brother after one week in isolation. Various researches such as Marxieh, S and Hadi, B (2017) discovered that visiting patients makes them excited and it reduces the duration of delivery. But this research concludes a different scenario in this case. The coincidence of the suicide scenery with a visit by his sister and brother provides an evidence that the deceased was afraid of piling a burden on others. It can also be concluded that he might have developed a sense of thwarted belongingness due to the inability to interact with his brother and sister despite having them in his own house. Consequently, the culprit must have surrendered his life in fear and empathy of infecting his relatives. The analysis of this research finding conforms to Thomas Joiner (2005) psychological theory of suicide.

\section{Conclusion}

There are many risk factors leading to suicide such as substance abuse, adverse life events, family history, a history of sexual abuse, troubled relationships, financial challenges, difficulties with sexual identity among others. In this case study, the effect evidence of troubled relationship. There is no history of suicide from the deceased family and this annuls the influence of nature. The fact that the deceased possessed a characteristic of quietness and reserved compounded with adverse life events brought about by coronavirus infection makes it a clear contributing factor.

There is also enough evidence that the deceased was afraid of losing his job and fear of infecting his relatives which yields altruism as proposed in the theory of suicide by Emile Durkheim (Lehmann, 1995). There is also evidence of reduced belongingness brought about coronavirus isolation requirements. Overall, this research concludes that the major risk factors to suicide are the things that people love so dearly and think life is worthless without those things.

Finally, the paper recommends that labor laws should protect private sector workers who are temporarily unavailable for an unknown period of time due to valid health reasons. Private sector enterprises are profit oriented and would not want to pay a worker who is idle for a prolonged period of time. But in circumstances where absenteeism is beyond the worker's control, the law should be clear to enable these people to resume their duties at some point. Sometimes laws are there but people pay little attention and it is very pathetic for skilled workers to be devoid of labor laws in the country they are working. This gives room for employees to manipulate such lazy, carefree and frivolous workers.

\section{References}

Baulch, Bob., Botha, Rosemary. and Pauw, Karl. (2020). The short-term impacts of COVID-19 on the Malawian economy, 2020-2021: A SAM multiplier modeling analysis. MaSSP Report November 2020. Washington, DC: International Food Policy Research Institute (IFPRI). https://doi.org/10.2499/p15738coll2.134160

Benfica, R., Squarcina, M. and de la Fuente, A. (2018) Structural transformation and poverty in Malawi: Decomposing the effects of occupation and spatial mobility. International Fund for Agricultural Development (IFAD) 
Centers for Disease Control and Prevention (2004). National Center for Health Statistics. Health, United States, 2004 with chartbook on trends in the health of Americans. Hyattsville, MD: Author.

Compton, M. and Berger, J. (2001). The Depressed Suicidal Patient. Annals of the New York Academy of Sciences. Retrieved from: https://www.researchgate.net/publication/285810615_The_Depressed_Suicidal_Patient. Accessed on 12 March 2021.

Curtin, S.C. and Heron, M. (2019), Death rates Due to Suicide and Homicide Among Persons Aged 10-24: United States, 2000 - 2007. Center for Disease Control and Prevention, National Center for Health Statistics. https://www.cdc.gov/ nchs/products/index.htm.

Griffiths, Mark. and Mamun, Mohammed. (2020). COVID-19 suicidal behavior among couples and suicide pacts: Case study evidence from press reports. Psychiatry Research. 289. e113105. 10.1016/j.psychres.2020.113105

Harmon, Vanessa., Mujkic, E., Kaukinen, C. and Weir, Henriikka. (2018). Causes and explanations of suicide terrorism: A systematic review. Homeland Security Affairs 14, Article 9 (December 2018). https://www.hsaj.org/articles/14749

Hill R.M., Rufino, K., Kurian, S., Saxena, J., Saxena, K. and Williams, L. (2020). Suicide ideation and attempts in a pediatric emergency department before and during COVID-19. Pediatrics. doi: 10.1542/peds.2020-029280

Joiner, T. (2009, June). The interpersonal-psychological theory of suicidal behavior: Current empirical status. Psychological Science Agenda. http://www.apa.org/science/about/psa/2009/06/sci-brief

Kathy, Stolley. S. (2005). The Basics of Sociology. London: Greenwood Press.

Kricheli, I., Rosner, Y., Mendelboim, A. and Schweitzer, Y. (2017). (Rep.). Institute for National Security Studies. Retrieved February 14, 2021, from http://www.jstor.org/stable/resrep08739

Lehmann, J. (1995). Durkheim\&\#39; s Theories of Deviance and Suicide: A Feminist Reconsideration. American Journal of Sociology, 100(4), 904-930. Retrieved 18 February 2021, from http://www.jstor.org/stable/2782155

Laanani, M. (2015). Impact of unemployment rate and the economic crisis on suicide mortality in Western European countries (2000-2010): Moussa Laanani, European Journal of Public Health, 25, Issue suppl_3, 51-52, ckv168.049, https://doi.org/10.1093/eurpub/ckv168.049

Rachiotis, G., Stuckler, D., McKee, M. and Hadjichrisodoulou, C. (2015). What has happened to suicides during the Greek economic crisis? Findings from an ecological study of suicides and their determinants (2003-2012) BMJ Open. 5, e007295. doi: 10.1136/bmjopen-2014-007295

Takahashi, Y. (2001). Depression and suicide. Japan Medical Association Journal, 44(8), 359-363.

Wilson, E. O. (1975). Some central problems of sociobiology. Social Science Information, 14(6), 5-18. doi: 10.1177/ 053901847501400601

Zortea, T.C., Brenna, C.T.A. and Joyce, M., et al. (2020). The impact of infectious disease-related public health emergencies on suicide, suicidal behavior, and suicidal thoughts: A systematic review. Crisis. 1-14. doi:10.1027/0227-5910/ a000753 pmid:33063542

Cite this article as: Lloyd GeorgeBanda (2021). A n investigation into Covid-19 related suiciderisk factors in M alawi. International Journal of A frican Studies. 1(2), 43-48. doi: 10.51483/ IJA FRS.1.2.2021.43-48. 